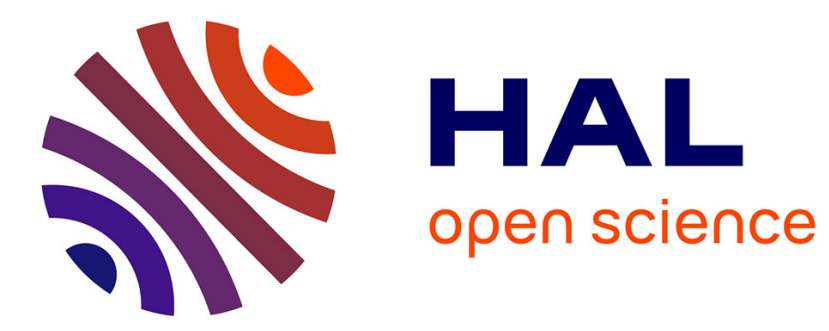

\title{
Parallelization of subdomain methods with overlapping for linear and nonlinear convection-diffusion problems
}

Pierre Spiteri, Ronan Guivarch, Didier El Baz, Chau Ming

\section{To cite this version:}

Pierre Spiteri, Ronan Guivarch, Didier El Baz, Chau Ming. Parallelization of subdomain methods with overlapping for linear and nonlinear convection-diffusion problems. 11-th International Conference on Parallel, Distributed and Network based Processing, Feb 2003, Gennes, Italy. pp.341-348, 10.1109/EMPDP.2003.1183609 . hal-01153602

\section{HAL Id: hal-01153602 \\ https://hal.science/hal-01153602}

Submitted on 20 May 2015

HAL is a multi-disciplinary open access archive for the deposit and dissemination of scientific research documents, whether they are published or not. The documents may come from teaching and research institutions in France or abroad, or from public or private research centers.
L'archive ouverte pluridisciplinaire HAL, est destinée au dépôt et à la diffusion de documents scientifiques de niveau recherche, publiés ou non, émanant des établissements d'enseignement et de recherche français ou étrangers, des laboratoires publics ou privés. 


\title{
Parallelization of Subdomain Methods with Overlapping for Linear and Nonlinear Convection-Diffusion Problems.
}

\author{
Pierre Spiteri' ${ }^{1}$, Ronan Guivarch', Didier El Baz², Chau Ming \\ 'ENSEEIHT-LIMA-IRIT \\ 2, rue Charles Camichel, 31071 Toulouse, France \\ ${ }^{2} \mathrm{LAAS}$ du CNRS \\ 7, avenue du Colonel Roche, 31077 Toulouse Cedex 4, France
}

\begin{abstract}
Linear and nonlinear convection-diffusion problems are considered. The numerical solution of these problems via the Schwarz alternating method is studied. A new class of parallel asynchronous iterative methods with flexible communication is applied. The implementation of parallel asynchronous and synchronous algorithms on distributed memory multiprocessors is described. Experimental results obtained on an IBM SP2 by using PVM are presented and analyzed. The interest of asynchronous iterative methods with flexible communication is clearly shown.
\end{abstract}

Keywords : Convection-diffusion problems, parallel asynchronous iterations, subdomain methods.

\section{Introduction}

Convection-diffusion problems occur in many domains such as hydraulics and finance. The discretization of such problems leads to the solution of very large scale systems of algebraic equations. In this context, the introduction of parallel algorithms via decomposition techniques can be very attractive. Domain decomposition methods and particularly the Schwarz alternating method are now well known. The purpose of this paper is the parallelization of subdomain methods with overlapping for the solution of linear and particular nonlinear convection-diffusion problems. More precisely, we study parallel asynchronous iterative schemes with flexible communication in connection with the Schwarz alternating method. The combination of such an asynchronous scheme with such a subdomain method tends to the behavior of a multiplicative Schwarz alternating method. The choice of the Schwarz alternating method comes from the observation that among subdomain methods, overlapping methods such as the Schwarz alternating methods are the most efficient for solving this kind of problems (see [7]). We recall that standard asynchronous algorithms are iterative methods whereby the components of the iteration vector are updated in parallel by several processors without any order nor synchronization (see [1] to [3], [8] and [9]). The restrictions imposed on asynchronous iterative algorithms are weak: no component of the iterate vector must be abandoned forever and more and more recent values of the components of the iterate vector have to be used as the computations are in progress.

The new class of asynchronous algorithms introduced in [10] is a general class of parallel iterative algorithms whereby very flexible communications are allowed. Contrarily to standard asynchronous iterative algorithms, whereby data exchange occur only at the end of each updating phase, asynchronous iterations with flexible communication permit one to consider the use of partial updates, i.e. the current value of the components of the iterate vector which is not labelled by an iteration number. Thus, there is a better coupling between communication and computation. We can expect a faster convergence and a reduction of computation time. The monotone convergence of asynchronous iterations with flexible communication can be proved in the theoretical context of partial ordering and discrete maxi- 
mum principle (see [10]). More precisely, if we consider the solution of the following algebraic system

$$
\mathcal{A}(U)=0
$$

where $\mathcal{A}$ is an $M$-function according to Rheinboldt (see [12]), we can show that if we initialize the algorithm by using a vector $U^{0}$ satisfying $\mathcal{A}\left(U^{0}\right) \geq 0$, then, under weak assumptions, the asynchronous iterative sequence converges monotonically to the solution $U^{*}$. Moreover, the solution of problem (1.1) by using the Schwarz alternating method leads to the solution of the following problem

$$
\widetilde{\mathcal{A}}(\widetilde{U})=0
$$

where $\widetilde{\mathcal{A}}$ is the increased system obtained from problem (1.1) (see [10]). We can easily verify that $\widetilde{\mathcal{A}}$ is also an $M$-function. Thus, the theoretical results obtained in the context of problem (1.1) can be applied to problem (1.2).

Asynchronous iterations with flexible communication have been applied to the solution of several classes of nonlinear boundary value problems in [13], where numerical experiments have been presented for a shared memory machine. Asynchronous iterations with flexible communication have also been applied to a particular class of convex optimization problems, i.e. network flow problems in [4]. The reader may refer to [5] for studies on nonlinear boundary value problems in the context of standard asynchronous iterations analyzed by using contraction techniques, see also [6]. If we discretize the diffusion operator by using a five points scheme and the convection operator by an appropriate one side backward or forward scheme, then we are in the framework of our study. It is one of the main goals of this paper to show that the operator which occurs in the discretized problem is an $M$-function and that monotone convergence can be shown. In this context, it is recommended to use decentered discretization schemes for the solution of the convection-diffusion equation in order to reduce numerical instability phenomena in the case of dominating convection. Indeed, computational experiments have shown that parallel iterative methods converge in a small number of iterations when a proper way of scanning the grid is used. In this case, it results from the discretization techniques and the difference of order of magnitude of the entries of the discretization matrix that this matrix is almost triangular. In this context we can solve the system on each subdomain by using a relaxation method which corresponds to a quasidirect method.

Section 2 deals with convection-diffusion problems. Asynchronous iterations with flexible communication are presented in section 3. The implementation and communication management of asynchronous algorithms with flexible communication on an IBM-SP2 is described in section 4 where computational results are also presented and analyzed.

\section{Convection-diffusion problems}

In this section, we consider linear and nonlinear convection-diffusion problems and we exhibit a general framework that will permit us in the sequel to show the convergence of parallel asynchronous algorithms with flexible communication. Consider the following linear convectiondiffusion problem

$$
\left\{\begin{array}{l}
-\nu \Delta u+a \frac{\partial u}{\partial x}+b \frac{\partial u}{\partial y}+c u=f, \text { everywhere in } \Omega \\
u=0, \text { on } \partial \Omega
\end{array}\right.
$$

where $c \geq 0, \nu>0, \Omega$ is the unit square and $f$ is a given function of $\mathcal{L}^{2}(\Omega)$. In the sequel $\partial \Omega$ will denote the boundary of $\Omega$. Consider now the discretization of the domain $\Omega$. In order to simplify the presentation we assume that the mesh is uniform. We denote by $h$ the stepsize. First, assume that the columns of the mesh are numbered according to the natural way. The discretization of the operators arising in problem (2.1) is made according to the following rules : the second derivative is discretized by the classical five points scheme, the first derivatives are discretized as follows according to the sign of $a$ and $b$. For example, we have

$$
\frac{\partial u}{\partial x}=\left\{\begin{array}{l}
\frac{u(x, y)-u(x-h, y)}{h}+\mathrm{O}(h), \text { if } a>0 \\
\frac{u(x+h, y)-u(x, y)}{h}+\mathrm{O}(h), \text { if } a<0 .
\end{array}\right.
$$

Proposition 1 Let $c$ be strictly positive and $A$ be the discretization matrix of problem (2.1). Then $A$ is an $M$-matrix.

Proof According to the discretization schemes defined by (2.2), we can verify by a simple calculus that the offdiagonal entries of matrix $A$ are nonpositive and the diagonal entries of $A$ are positive whatever the sign of $a$ and $b$. Thus, $A$ is a $Z$-matrix. Furthermore, $A$ is strictly diagonally dominant. 
In the case where $c=0$, we can verify by the same technique that $A$ is an irreducibly diagonally dominant $Z$ matrix whatever the sign of $a$ and $b$. Thus, $A$ is an $M$ matrix.

Consider now a red-black ordering of the columns and denote by $\widetilde{A}$, the corresponding discretization matrix.

Proposition 2 Let $c$ be strictly positive. Then $\widetilde{A}$ is an $M$ matrix.

Proof For the discretization considered, $\widetilde{A}$ is clearly a $Z$ matrix; moreover $\widetilde{A}$ is also strictly or irreducibly diagonally dominant. Thus, $\widetilde{A}$ is an $M$-matrix.

We present now various nonlinear convection-diffusion problems. The first application concerns a boundary temperature control problem modeled by the following equation

$$
\left\{\begin{array}{l}
-\nu \Delta u+a \frac{\partial u}{\partial x}+b \frac{\partial u}{\partial y}+c u=f \text { everywhere in } \Omega \\
\frac{\partial u}{\partial n}+\varphi(u)=0 \text { on } \Gamma_{d} \text { and } u=0 \text { on } \partial \Omega-\Gamma_{d}
\end{array}\right.
$$

where $\Omega \subset \mathbb{R}^{2}$ (or $\mathbb{R}^{3}$ ), $c \geq 0, \Gamma_{d} \subset \partial \Omega, f \in \mathcal{L}^{2}(\Omega)$ and $\varphi: \mathbb{R} \rightarrow \mathbb{R}$ is a continuous nondecreasing function. In the sequel, we will consider for $\varphi$, the graphs displayed in figure 2.1.

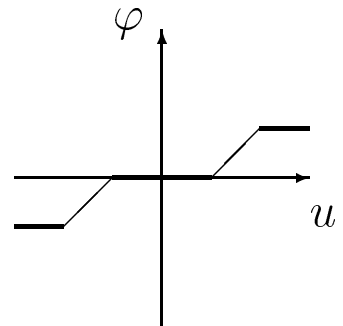

(a)

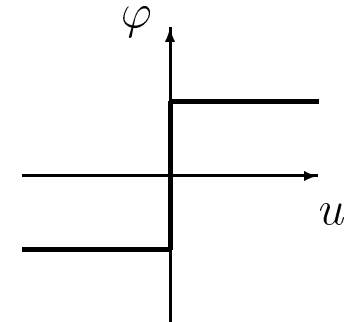

(b)
Figure 2.1. Different graphs for $\varphi$

Note that the second graph models a multi-valued function corresponding to the following boundary condition : $\frac{\partial u}{\partial n}+\varphi(u) \ni 0$. The discretization techniques presented above can be used for the interior points of domain $\Omega$. The discretization of the Neumann condition leads to the solution of the following discrete equations

$$
\frac{u_{j}-u_{j-1}}{h}+\varphi\left(u_{j}\right)=0,
$$

for all discretization points in $\Gamma_{d}$. We have to solve the discretized system (1.1) where

$$
\mathcal{A}(U)=A U+\phi(U)-g,
$$

and $A$ is the discretization matrix associated with the natural block ordering relative to the linear part, $\phi$ is a diagonal nondecreasing operator and $(g, u) \in \mathbb{R}^{\operatorname{dim}(A)} \times \mathbb{R}^{\operatorname{dim}(A)}$.

According to (2.4), note that the components of $\phi$ are null for the interior points of $\Omega$ and equal to $h \varphi\left(u_{j}\right)$ if $j$ corresponds to the index of a point which belongs to $\Gamma_{d}$.

Proposition $3 A$ is an $M$-matrix.

Proof If $c>0$, then it follows from (2.4), Proposition 1 and the Dirichlet condition defined on $\partial \Omega-\Gamma_{d}$ that $A$ is a strictly diagonally dominant $Z$-matrix. Thus, $A$ is an $M$-matrix. If $\mathrm{c}=0$, then the matrix $A$ is clearly diagonally dominant. Furthermore, by using the characterization of irreducible matrices (see [11]), we can verify that $A$ is irreducible diagonally dominant.

In order to deal with the nonlinearity, we introduce now the following standard terminology (see [11] and [12]). The mapping $\mathcal{A}$ is an $M$-function if $\mathcal{A}$ is off-diagonally antitone and inverse isotone.

Proposition 4 The mapping $\mathcal{A}$ defined by (2.5) is an $M$ function.

Proof We recall that $A$ is an $M$-matrix and $\phi$ is a continuous nondecreasing diagonal mapping. Then, it follows from Theorem 13.5.6 of [11] that $\mathcal{A}$ is an $M$-function.

The above results can be extended to the case where a red black ordering is considered.

Rather than considering a boundary value problem with mixed conditions, we can study a problem with Neumann conditions defined everywhere on $\partial \Omega$. Then, the previous analysis still holds if the condition $c>0$ is satisfied. We turn now to the study of problems with different kinds of nonlinearities which are defined on $\Omega$ rather than $\partial \Omega$. The general model can be given as follows

$$
\left\{\begin{array}{l}
-\nu \Delta u+a \frac{\partial u}{\partial x}+b \frac{\partial u}{\partial y}+c u+\varphi(u)=f \text { in } \Omega, \\
\text { B.C. }
\end{array}\right.
$$

where $c>0, f \in \mathcal{L}^{2}(\Omega), \varphi: \mathbb{R} \rightarrow \mathbb{R}$ is a continuous nondecreasing function and B.C. represents the classical boundary conditions (i.e. Dirichlet, Neumann, Fourier 
and mixed). By using the discretization techniques quoted above, we obtain an operator similar to (2.5) and we are in the general framework of $M$-functions. Among the many nonlinear functions that we can consider, we quote for example : $\varphi(u)=e^{\alpha u}$, with $\alpha>0, \varphi(u)=\log (\beta+\delta u)$, with $\delta>0$ and a convenient sign for $\beta$. Another very interesting application concerns the obstacle problem.

$$
\left\{\begin{array}{l}
\text { Find } u \in \mathrm{K} \text { such that } \\
b(u, v-u) \geq(f, v-u), \text { for all } v \in \mathrm{K}
\end{array}\right.
$$

where $\mathrm{K}$ is the convex closed set defined as follows

$$
\mathrm{K}=\left\{v \in \mathrm{H}_{0}^{1}(\Omega) \mid v \leq \psi \text { everywhere in } \Omega\right\}
$$

$b($.$) is the classical continuous nonsymmetric bilinear form$ associated with an elliptic operator $\mathcal{B}$ and $f \in \mathcal{L}^{2}(\Omega)$. Problem (2.7) can be formulated equivalently as follows

$$
\left\{\begin{array}{l}
\text { Find } u \in \mathrm{K} \text { such that } \\
0 \in \mathcal{B} u-f+\partial \chi_{\mathrm{K}}(u),
\end{array}\right.
$$

where $\partial \chi_{\mathbf{K}}(u)$ is the sub-differential of the indicator function $\chi_{\mathrm{K}}$ of the convex set $\mathrm{K}$ whose graph is given by figure 2.2. Clearly $\partial \chi_{\mathrm{K}}$ is monotone nondecreasing.

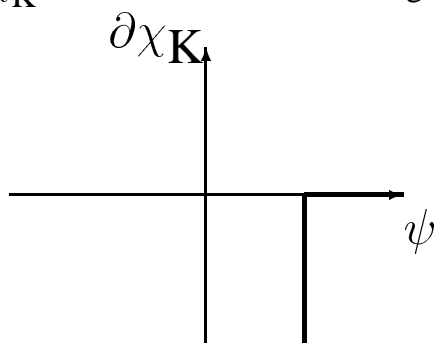

Figure 2.2. Graph of $\partial \chi_{\mathbf{K}}$

\section{Asynchronous iterations}

Let $\mathcal{A}$ be a continuous surjective $M$-function from $\mathbb{R}^{n}$ onto $\mathbb{R}^{n}$. We consider the solution of the system of equations (1.1). Under the above assumptions, (1.1) has a unique solution $U^{*}$. Let $\mathbb{R}^{n}$ be denoted by $E$ and consider the following decomposition of $E, E=\prod_{i=1}^{\alpha} E_{i}$, where $\alpha$ is a positive integer, $E_{i}=\mathbb{R}^{n_{i}}$, and $\sum_{i=1}^{\alpha} n_{i}=n$. Each subspace $E_{i}$ is endowed with the natural partial ordering, i.e. component by component. Let $W \in E$ and consider the following splitting of $W: W=\left\{W_{1}, \ldots, W_{i}, \ldots, W_{\alpha}\right\} \in$ $\prod_{i=1}^{\alpha} E_{i}$ and the corresponding block-decomposition of $\mathcal{A}(W): \mathcal{A}(W)=\left\{\mathcal{A}_{1}(W), \ldots, \mathcal{A}_{i}(W), \ldots, \mathcal{A}_{\alpha}(W)\right\} \in$ $\prod_{i=1}^{\alpha} E_{i}$. For all $i \in\{1, \ldots, \alpha\}$, and $W \in E$, we introduce the following mapping.

$V_{i} \rightarrow \mathcal{A}_{i}\left(V_{i} ; W\right)=\mathcal{A}_{i}\left(W_{1}, \ldots, W_{i-1}, V_{i}, W_{i+1}, \ldots, W_{\alpha}\right)$

Since $\mathcal{A}$ is a continuous, surjective $M$-function, it follows from the Theorem 3.5 of [12] that the mapping $V_{i} \longrightarrow$ $\mathcal{A}_{i}\left(V_{i} ; W\right)$ is a continuous surjective $M$-function from $E_{i}$ into $E_{i}$, for all $i \in\{1, \ldots, \alpha\}$ and $W \in E$. Moreover, for all $i \in\{1, \ldots, \alpha\}$ and $W \in E$, the system

$$
\mathcal{A}_{i}\left(U_{i} ; W\right)=0
$$

has a unique solution $\widehat{U}_{i}$. Hence, we can define a fixed point mapping $F: E \rightarrow E$, associated with problem (1.1) such that

$$
F(W)=\widehat{U}=\left\{\widehat{U}_{1}, \ldots, \widehat{U}_{i}, \ldots, \widehat{U}_{\alpha}\right\}
$$

The mapping $F$ is univoquely defined and it can be proved that $F$ is monotone nondecreasing on $E$ (see [12]).

The following concept permits one to define a set of starting points for the parallel asynchronous iterative algorithms.

Definition 1 Every $V \in \mathbb{R}_{+}^{n}$ is an $\mathcal{A}$-super-solution if $\mathcal{A}(V) \geq 0$.

Throughout the paper, asynchronous sequences are studied by using the concept of order intervals which is defined as follows: let $\left(V_{i}, V_{i}^{\prime}\right) \in E_{i}^{2}$ such that $V_{i} \leq V_{i}^{\prime}$, the order interval $\left\langle V_{i}, V_{i}^{\prime}\right\rangle_{i}=\left\{W_{i} \in E_{i} \mid V_{i} \leq W_{i} \leq V_{i}^{\prime}\right\}$. Similarly, let $\left(V, V^{\prime}\right) \in E^{2}$ such that $V \leq V^{\prime},\left\langle V, V^{\prime}\right\rangle=$ $\left\{W \in E \mid V \leq W \leq V^{\prime}\right\}$. We introduce now classes of fixed point mappings which play important parts in the definition of asynchronous iterative methods with flexible communication.

Definition 2 Let $\mathcal{A}$ be an $M$-function. $F^{\mathcal{A}}$ is an $\mathcal{A}$ supermapping associated with $F$ if for all $i \in\{1, \ldots, \alpha\}$ and $V \in E$ such that $\mathcal{A}_{i}(V) \geq 0, F_{i}^{\mathcal{A}}(V) \in E_{i}$ is such that $F_{i}^{\mathcal{A}}(V) \leq V_{i}, \mathcal{A}_{i}\left(F_{i}^{\mathcal{A}}(V) ; V\right) \geq 0$ and $F_{i}^{\mathcal{A}}(V) \neq V_{i}$ if $F_{i}(V) \neq V_{i}$.

Definition $3 F^{\mathcal{A}}$ is an $\mathcal{M}$-continuous $\mathcal{A}$-supermapping associated with $F$ if there exists an $\mathcal{A}$-supermapping $F^{\mathcal{B}}$ associated with $F$ such that $F_{i}^{\mathcal{B}}(V) \in\left\langle F_{i}^{\mathcal{A}}(V), V_{i}\right\rangle_{i}$ for all 
$i \in\{1, \ldots, \alpha\}$ and $V \in E$ such that $\mathcal{A}_{i}(V) \geq 0$, and $V^{p} \downarrow V^{*}, p \rightarrow \infty$, implies $F_{i}^{\mathcal{B}}\left(V^{p}\right) \downarrow F_{i}^{\mathcal{B}}\left(V^{*}\right), p \rightarrow \infty$, $i=1, \ldots, \alpha$, where $V^{p} \downarrow V^{*}$ means $V^{*} \leq \cdots \leq V^{p+1} \leq$ $V^{p} \leq \cdots \leq V^{0}$ and $\lim _{p \rightarrow \infty} V^{p}=V^{*}$.

The following concepts are central in the study of asynchronous iterations. A steering of block components of the iterate vector is a sequence $\mathcal{S}=\{s(p)\}, p \in \mathbb{N}$, such that $s(p) \in\{1, \ldots, \alpha\}$, for all $p \in \mathbb{N}$. We introduce now the mappings $\rho_{i}: \mathbb{N} \rightarrow \mathbb{N}, \forall i \in\{1, \ldots, \alpha\}$, such that for all $p \in \mathbb{N}, 0 \leq \rho_{i}(p) \leq p$, and $\rho_{i}(p)=p$ if $i=s(p)$. Hence, we define the mapping $\rho: \mathbb{N}^{\alpha} \rightarrow \mathbb{N}^{\alpha}$ with components $\rho_{i}(p), i \in\{1, \ldots, \alpha\}$ and the sets $K_{i}^{p}=\{k \in N \mid s(k)=$ $i, 0 \leq k<p\}, i \in\{1, \ldots, \alpha\}, p \in \mathbb{N}$.

Definition 4 The general class of asynchronous iterative methods with flexible communication $\left\{V^{p}\right\}$ is given as follows for all $p \in \mathbb{N}$ and $i \in\{1, \ldots, \alpha\}$,

$$
\left\{\begin{array}{l}
V_{i}^{p+1}=F_{i}^{\mathcal{A}}\left(\tilde{V}^{p}\right) \text { if } i=s(p), \\
V_{i}^{p+1}=V_{i}^{p} \text { if } i \neq s(p),
\end{array}\right.
$$

where $F^{\mathcal{A}}$ is an $\mathcal{A}$-supermapping associated with $F$,

$$
\begin{gathered}
\widetilde{V}^{0}=V^{0} \text { is an } \mathcal{A} \text {-super-solution, } \\
\widetilde{V}^{p} \in\left\langle V^{p}, \min \left(V^{\rho(p)}, \widetilde{V}^{q}\right)\right\rangle \text { if } p \geq 1,
\end{gathered}
$$

$V^{\rho(p)}$ denotes the vector of $E$ with block components $V_{i}^{\rho_{i}(p)}, i \in\{1, \ldots, \alpha\}$ and $q=\operatorname{Max}\left\{k \in K_{s(p)}^{p}\right\}$.

Asynchronous iterative algorithms with flexible communication defined recursively by (3.3) to (3.5) are general iterative methods whereby iterations are carried out in parallel by up to $\beta$ processors without any order nor synchronization $(\beta \leq \alpha)$. The main feature of this class of iterative methods is to allow very flexible communication or data exchange between the processors. In a typical update, a component $\widetilde{V}_{j}^{p}$ of the iterate vector is taken anywhere in the order interval $\left\langle V_{j}^{p}, \min \left(V_{j}^{\rho_{j}(p)}, \widetilde{V}_{j}^{q}\right)\right\rangle_{j}$, where $\widetilde{V}_{j}^{q}$ was the value used in the last update of the same block component and $V_{j}^{\rho_{j}(p)}$ models the nondeterministic behavior of the iterative scheme. Thus, the value of the components of the iterate vector which is used in an update may come from updates which are in progress. Processors can exchange the current value of the relaxed components at any time (see figure 3.1, where processors are denoted by $P_{1}$ and $P_{2}$ and rectangles correspond to updating phases i.e. iterations). On the other hand, all processors can use the most recent values of the components of the iterate vector.

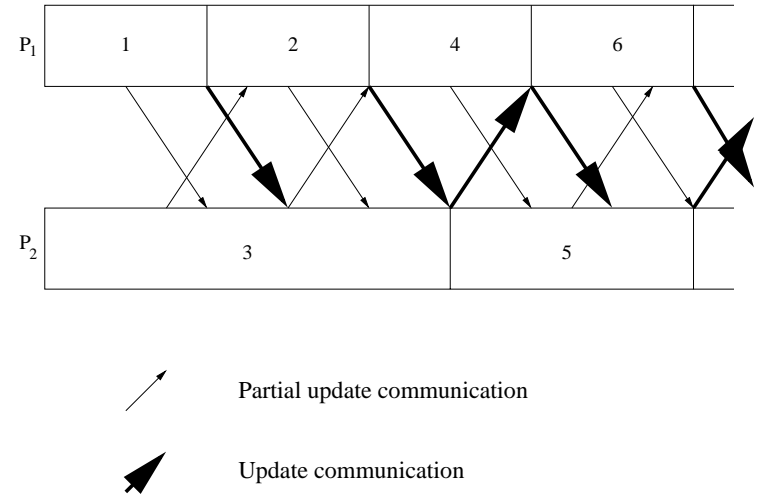

Figure 3.1. Example of flexible communications between two processors.

Proposition 5 (see [10]) Let $\mathcal{A}$ be a continuous surjective $M$-function, $F$ the fixed point mapping associated with $\mathcal{A}$ defined by (3.1) and (3.2), $F^{\mathcal{A}}$ an $\mathcal{M}$-continuous $\mathcal{A}$ supermapping associated with $F, V^{0} \in E$ an $\mathcal{A}$-supersolution. Assume that the steering $\mathcal{S}$ satisfies

$$
\{p \in \mathbb{N} \mid i \in s(p)\} \text { is infinite } \forall i \in\{1, \ldots, \alpha\}
$$

and assume also that we have

$$
\lim _{p \rightarrow \infty} \rho_{i}(p)=+\infty, \text { for all } i \in\{1, \ldots, \alpha\}
$$

Then, asynchronous iterations with flexible communication $\left\{V^{p}\right\}$ given by (3.3) to (3.5) are well defined and converge monotonically to $V^{*}$ the unique solution of problem (1.1).

We concentrate in the sequel on the Schwarz alternating method which is commonly used for the solution of boundary value problems. This method is also well suited to parallel computation. In order to illustrate the coupling between the Schwarz alternating method and asynchronous iterations with flexible communication, we consider the linear problem (2.1). This problem can be decomposed into $\alpha$ subproblems as follows

$$
\left\{\begin{array}{l}
-\nu \Delta u_{i}+a \frac{\partial u_{i}}{\partial x}+b \frac{\partial u_{i}}{\partial y}+c u_{i}=f_{i}, \text { everywhere in } \Omega_{i}, \\
u_{i / \Gamma_{i}}=0, \\
u_{i / \gamma_{i}^{1}}=u_{i-1 / \gamma_{i}^{1}} \text { for } 2 \leq i \leq \alpha, \\
u_{i / \gamma_{i}^{2}}=u_{i+1 / \gamma_{i}^{2}} \text { for } 1 \leq i \leq \alpha-1 .
\end{array}\right.
$$


where $u_{i}$ and $f_{i}$, respectively, are the restriction of $u$ and $f$, respectively, to $\Omega_{i}$ and $\Omega=\bigcup_{i=1}^{\alpha} \Omega_{i}, \Omega_{i} \cap \Omega_{i+1} \neq \emptyset$, $\gamma_{i}^{1}=\partial \Omega_{i} \bigcap \Omega_{i-1}, i \in\{2, \ldots, \alpha\}, \gamma_{i}^{2}=\partial \Omega_{i} \bigcap \Omega_{i+1}, i \in$ $\{1, \ldots, \alpha-1\}, \Gamma_{i}=\partial \Omega_{i} \bigcap \partial \Omega$, (see figure 3.2). The de-

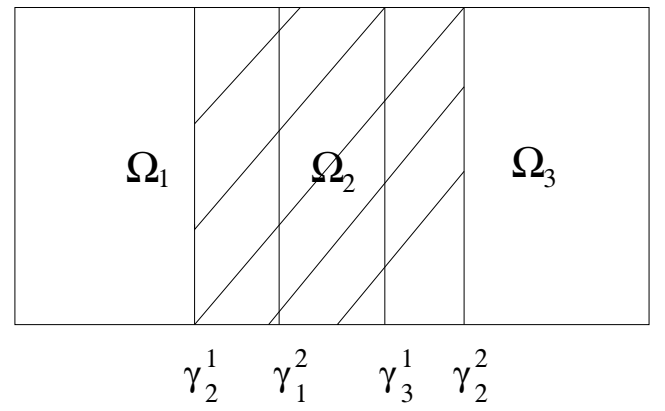

Figure 3.2. An example with 3 subdomains.

composition (3.8) corresponds to a subdomain decomposition with overlapping whereby $u_{i}$ is computed by using the restriction of $u_{i-1}$ and $u_{i+1}$ on $\gamma_{i}^{1}$ and $\gamma_{i}^{2}$, respectively. In the sequential case, the scheme of computation corresponds exactly to the multiplicative Schwarz method. In order to be as close as possible to the multiplicative scheme, the parallel version considered here combines the Schwarz method with an asynchronous iterative scheme with flexible communication. According to (3.3) to (3.5), if $i \in s(p)$, then the value of the iterate vector $u_{i}^{p}$ is computed as follows

$\left\{\begin{array}{l}-\nu \Delta u_{i}^{p}+a \frac{\partial u_{i}^{p}}{\partial x}+b \frac{\partial u_{i}^{p}}{\partial y}+c u_{i}^{p}=f_{i}, \text { everywhere in } \Omega_{i}, \\ u_{i / \Gamma_{i}}^{p}=0, \\ u_{i / \gamma_{i}^{1}}^{p}=w_{i-1 / \gamma_{i}^{1}}, \text { for } 2 \leq i \leq \alpha, \\ u_{i / \gamma_{i}^{2}}^{p}=w_{i+1 / \gamma_{i}^{2}}, \text { for } 1 \leq i \leq \alpha-1 .\end{array}\right.$

where $w_{i-1 / \gamma_{i}^{1}}$ and $w_{i+1 / \gamma_{i}^{2}}$, respectively, denote the available value of $u_{i-1}$ and $u_{i+1}$ on $\gamma_{i}^{1}$ and $\gamma_{i}^{2}$, respectively. Note that any constant $u^{0}$ satisfying $\frac{f}{c} \leq u^{0}$, is an initial $\mathcal{A}$-super-solution guess. We consider an $M$-function $\mathcal{A}$, obtained by a diagonal monotone perturbation $\varphi$ of an $M$-matrix $A$. Thus, we have a problem similar to problem (2.5). We study the solution of the following nonlinear simultaneous equations: $\mathcal{A}(U)=0$, via an asynchronous subdomain method derived from the Schwarz alternating method. The augmentation process of the Schwarz alternating method transforms the matrix $A$ into a matrix $\widetilde{A}$ which is also an $M$-matrix and the nonlinear mapping $\varphi$ into the monotone increasing mapping $\widetilde{\varphi}$ (see [13]). Thus, the resulting nonlinear mapping $\widetilde{\mathcal{A}}$ is a surjective $M$-function and

the above study can be applied. The problem can be solved on each subdomain by using any direct or iterative method. In the case where $\nu=0$, the discretization matrix is triangular. Thus, a relaxation method converges in only one iteration when the scanning order of the grid matches the triangular discretization matrix. In the case where $\nu$ is small, the discretization matrix is nearly triangular, as a matter of fact it results from the decentered discretization scheme that the entries relative to a triangular part of the matrix are an order of magnitude greater than the entries of the other part which are equal to $\nu$. The use of the relaxation method on each subdomain then corresponds to a quasi direct method with low computation cost.

\section{Numerical Experiments}

Asynchronous iterative algorithms with flexible communication were carried out on an I.B.M-SP2 distributed memory multiprocessor of CINES. Each processor is a Power2 and has a peak performance of 266 M-Flops and 520 Megabytes of RAM. The processors are connected via a multi-level Omega network. Processors are dedicated to the execution of a given task, the switch is shared by the different users of the parallel machine. The communications are performed by using PVM Asynchronous algorithms were carried out according to the following "hostcustomer" model.

Host:

\section{while non global convergence do : \\ o computation. \\ o global convergence test : nonblocking receipt of convergence test of the customers.}

done

- broadcast the global convergence message to the customers.

- Receipt of the solution of each subdomain from the customers. end

The host activates all other processors, performs computations on one or several subdomains and is dedicated to the termination detection of the Schwarz alternating method. Each customer performs computations on one or several

while non global convergence do :

- Nonblocking receipt of the global convergence message from the master processor.

o computation.

$\circ \overline{\text { broadcast the convergence }}$ test to the host. done

- broadcast the solution of each subdomain treated. subct end

\section{$\underline{\text { Customers: }}$}


subdomains. The general algorithm can be described as follows.

The efficiency of parallel algorithms strongly depends on the communication frequency within the computations. The following algorithm describes the way flexible communications are performed.

High Communication Frequency (HCF):

procedure computation

○ for each subdomain

do

- Gauss-Seidel relaxation.

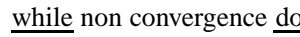

- Nonblocking receipt of the interaction values

from the neighbors.

- Relaxation.

- Broadcast the interaction values to the neighbors. done.

done

The stopping criterion is given as follows $\sup _{i} \mid u_{i}^{p+1}-$ $u_{i}^{p} \mid \leq \epsilon$, where $\epsilon$ is a chosen accuracy. We note also that if we start simultaneously from an $\mathcal{A}$-supersolution and from an $\mathcal{A}$-subsolution, the solution of the problem will be upperbounded and lowerbounded. Thus in this context, we will have a reliable stopping criterion. The host performs the termination test very fast. As a matter of fact, the test is a simple logical operation which follows the communication of local termination test of customers.

\begin{tabular}{|c|c|c|c|c|}
\hline Method & $\mathrm{r}$ & $\mathrm{t}$ & $\mathrm{s}$ & $\mathrm{e}$ \\
\hline $\mathrm{Se}$ & 77481 & 1986.3 & - & - \\
\hline $\mathrm{S} 2$ & 81659 & 1136.8 & 1.74 & 0.87 \\
\hline $\mathrm{A} 2$ & 77347 & 1002.9 & 1.98 & 0.99 \\
\hline $\mathrm{S} 4$ & 89410 & 660.8 & 3.00 & 0.75 \\
\hline $\mathrm{A} 4$ & 79606 & 553.8 & 3.58 & 0.90 \\
\hline
\end{tabular}

Table 4.1. Problem (2.1): $\nu=1,8$ subdomains.

In the sequel, the sequential method is denoted by $S e$. Parallel synchronous and asynchronous algorithms are denoted by $\mathrm{Sx}$ and Ax, respectively, where $\mathrm{x}$ represents the number of processors. The total number of Gauss-Seidel relaxation steps is denoted by $r$. The elapsed time is denoted by $t ; s$ and $e$, respectively, denote the speedup and efficiency, respectively. Tables 4.1 to 4.3 display the performance of sequential and parallel Schwarz alternating al-

\begin{tabular}{|c|c|c|c|c|}
\hline Method & $\mathrm{r}$ & $\mathrm{t}$ & $\mathrm{s}$ & $\mathrm{e}$ \\
\hline $\mathrm{Se}$ & 83555 & 2084.9 & - & - \\
\hline $\mathrm{S} 2$ & 91514 & 1245.2 & 1.68 & 0.84 \\
\hline $\mathrm{A} 2$ & 91732 & 1175.2 & 1.78 & 0.89 \\
\hline $\mathrm{S} 4$ & 106547 & 797.1 & 2.62 & 0.66 \\
\hline A4 & 95267 & 666.0 & 3.13 & 0.78 \\
\hline
\end{tabular}

Table 4.2. Problem (2.1): $\nu=0.1,8$ subdomains,

\begin{tabular}{|c|c|c|c|c|}
\hline Method & $\mathrm{r}$ & $\mathrm{t}$ & $\mathrm{s}$ & $\mathrm{e}$ \\
\hline $\mathrm{Se}$ & 12382 & 314.1 & - & - \\
\hline $\mathrm{S} 2$ & 13895 & 198.3 & 1.58 & 0.79 \\
\hline $\mathrm{A} 2$ & 13486 & 180.0 & 1.75 & 0.87 \\
\hline $\mathrm{S} 4$ & 17037 & 126.2 & 2.49 & 0.62 \\
\hline $\mathrm{A} 4$ & 15201 & 113.5 & 2.76 & 0.69 \\
\hline
\end{tabular}

Table 4.3. Problem (2.1): $\nu=0.01,8$ subdomains

gorithms for linear problem (2.1) with 130305 discretization points, 8 subdomains and different values of the diffusion coefficient. We consider $\nu=1, \nu=0.1$ and $\nu=0.01$, respectively. Table 4.4 shows the performance of the same algorithms for problem (2.1) with 92837 discretization points, $\nu=1$ and 16 subdomains. Tables 4.5 to 4.7 display elapsed time, speed up and efficiency of sequential and parallel Schwarz alternating algorithms for nonlinear problem (2.3) with 130305 discretization points, 8 subdomains, $\nu=1, \nu=0.1$ and $\nu=0.01$, respectively. In all cases, $a=0.5, b=1.5$ and $c=10$. We note that according to the pattern of the discretization matrix, especially in the dominating convection case, we have used the proper way of scanning the grid with the Gauss-Seidel relaxation method. In this case, we have obtained good elapsed times; as an example we have obtained an elapsed time equal to 314.1 seconds for the sequential solution of linear problem (2.1) in table 4.3, with the proper way of scanning the grid and 398.3 seconds for the same solution with a different way of scanning the grid. From tables 4.1 to 4.7 , it can be seen that the performance of parallel asynchronous algorithms with flexible communication is better than the performance of synchronous algorithms. The lack of synchronization point and the use of the current value of the components of the iteration vector speeds up the convergence of the Schwarz alternating method. We note also that 


\begin{tabular}{|c|c|c|c|c|}
\hline Method & $\mathrm{r}$ & $\mathrm{t}$ & $\mathrm{s}$ & $\mathrm{e}$ \\
\hline $\mathrm{S}$ & 90204 & 839.4 & - & - \\
\hline S2 & 86194 & 437.1 & 1.92 & 0.96 \\
\hline A2 & 83589 & 396.5 & 2.12 & 1.06 \\
\hline S4 & 91820 & 244.3 & 3.44 & 0.86 \\
\hline A4 & 85366 & 227.0 & 3.70 & 0.93 \\
\hline S8 & 100613 & 146.0 & 5.75 & 0.72 \\
\hline A8 & 85302 & 115.9 & 7.25 & 0.91 \\
\hline
\end{tabular}

Table 4.4. Problem (2.1): $\nu=1,16$ subdomains

\begin{tabular}{|c|c|c|c|c|}
\hline Method & $\mathrm{r}$ & $\mathrm{t}$ & $\mathrm{s}$ & $\mathrm{e}$ \\
\hline $\mathrm{Se}$ & 85345 & 2147.7 & - & - \\
\hline $\mathrm{S} 2$ & 93306 & 1347.5 & 1.59 & 0.80 \\
\hline $\mathrm{A} 2$ & 88857 & 1247.4 & 1.72 & 0.86 \\
\hline $\mathrm{S} 4$ & 101708 & 761.2 & 2.82 & 0.71 \\
\hline $\mathrm{A} 4$ & 91440 & 637.7 & 3.37 & 0.84 \\
\hline
\end{tabular}

Table 4.5. Problem (2.3): $\nu=1,8$ subdomains

when the number of processors increases, the efficiency of synchronous algorithms decreases faster than the efficiency of asynchronous algorithms. Parallel algorithms are more efficient if two or more subdomains are assigned to each processor. Finally, the algorithms are not really sensitive to the size of the overlapping.

Acknowledgments : Part of this study was made possible by a grant of CINES, Montpellier, France.

\section{References}

[1] G. M. Baudet, Asynchronous iterative methods for multiprocessors, J.A.C.M., 25:226-244, 1978.

[2] D. P. Bertsekas and J. Tsitsiklis, Parallel and Distributed Computation, Numerical Methods, Englewood cliffs, Prentice Hall, 1989.

[3] D. Chazan and W. Miranker, Chaotic relaxation, Linear Algebra Appl., 2:199-222, 1969.

[4] D. El Baz, P. Spiteri, J.C. Miellou and D. Gazen, Asynchronous iterative algorithms with flexible communication for nonlinear network flow problems, Journal of Parallel and Distributed Computing, 38:1-15, 1996.

[5] L. Giraud and P. Spiteri, Résolution parallèle de problèmes aux limites non-linéaires, M.2 A.N., 25:73-100, 1991.

\begin{tabular}{|c|c|c|c|c|}
\hline Method & $\mathrm{r}$ & $\mathrm{t}$ & $\mathrm{s}$ & $\mathrm{e}$ \\
\hline $\mathrm{Se}$ & 32961 & 831.1 & - & - \\
\hline $\mathrm{S} 2$ & 35684 & 517.1 & 1.61 & 0.81 \\
\hline $\mathrm{A} 2$ & 33625 & 473.3 & 1.76 & 0.88 \\
\hline $\mathrm{S} 4$ & 40763 & 331.2 & 2.51 & 0.63 \\
\hline $\mathrm{A} 4$ & 38975 & 278.4 & 2.99 & 0.75 \\
\hline
\end{tabular}

Table 4.6. Problem (2.3): $\nu=0.1,8$ subdomains

\begin{tabular}{|c|c|c|c|c|}
\hline Method & $\mathrm{r}$ & $\mathrm{t}$ & $\mathrm{s}$ & $\mathrm{e}$ \\
\hline $\mathrm{Se}$ & 5974 & 152.6 & - & - \\
\hline $\mathrm{S} 2$ & 6401 & 97.4 & 1.57 & 0.78 \\
\hline $\mathrm{A} 2$ & 6415 & 91.6 & 1.67 & 0.84 \\
\hline $\mathrm{S} 4$ & 7305 & 62.2 & 2.45 & 0.61 \\
\hline $\mathrm{A} 4$ & 7500 & 57.7 & 2.65 & 0.66 \\
\hline
\end{tabular}

Table 4.7. Problem (2.3): $\nu=0.01,8$ subdomains

[6] R. Guivarch, Résolution parallèle de problèmes aux limites couplés par des méthodes de sous-domaines synchrones et asynchrones, Diplôme de Doctorat en Informatique de l'Institut National Polytechnique de Toulouse, 1997.

[7] K.H. Hoffmann and J. Zou, Parallel efficiency of domain decomposition methods, Parallel Computing, 19:1375-1391, 1993.

[8] J. C. Miellou, Itérations chaotiques à retards, étude de la convergence dans le cas d'espaces partiellement ordonnés C.R.A.S., Paris, 280:233-236, 1975.

[9] J. C. Miellou, Algorithmes de relaxation chaotiques à retard, RAIRO R1, 55-82, 1975.

[10] J. C. Miellou, D. El Baz and P. Spiteri, A new class of asynchronous iterative algorithms with order interval, Mathematics Of Computation, 67:237-255, 1998.

[11] J. M. Ortega and W. C. Rheinboldt, Iterative solution of nonlinear equations in several variables, Academic Press, New York, 1970.

[12] W. C. Rheinboldt, On $M$-functions and their application to nonlinear Gauss-Seidel iterations and to network flows, $J$. Math. Anal. and Appl., 32:274-307, 1970.

[13] P. Spiteri, J. C. Miellou and D. El Baz, Asynchronous Schwarz alternating method with flexible communication for the obstacle problem, Calculateurs Parralèles, Réseaux et Systèmes Répartis, 13, 2001. 